\title{
High- $Q$ Quasibound States in the Continuum for Nonlinear Metasurfaces
}

\author{
Zhuojun Liu, ${ }^{1}$ Yi Xu, ${ }^{2, *}$ Ye Lin, ${ }^{2}$ Jin Xiang, ${ }^{3}$ Tianhua Feng, ${ }^{2}$ Qitao Cao, ${ }^{4}$ Juntao Li, ${ }^{1}$ Sheng Lan, ${ }^{3}$ and Jin Liu ${ }^{1, \dagger}$ \\ ${ }^{1}$ State Key Laboratory of Optoelectronic Materials and Technologies, School of Physics, \\ Sun Yat-sen University, Guangzhou 510275, China \\ ${ }^{2}$ Department of Electronic Engineering, College of Information Science and Technology, \\ Jinan University, Guangzhou 510632, China \\ ${ }^{3}$ School of Information and Optoelectronic Science and Engineering, \\ South China Normal University, Guangzhou 510006, China \\ ${ }^{4}$ State Key Laboratory for Artificial Microstructures and Mesoscopic Physics, School of Physics, \\ Peking University, Beijing 100871, China
}

(Received 25 July 2019; revised manuscript received 5 September 2019; published 17 December 2019)

Sharp electromagnetic resonances play an essential role in physics in general and optics in particular. The last decades have witnessed the successful developments of high-quality $(Q)$ resonances in microcavities operating below the light line, which however is fundamentally challenging to access from free space. Alternatively, metasurface-based bound states in the continuum (BICs) offer a complementary solution of creating high- $Q$ resonances in devices operating above the light line, yet the experimentally demonstrated $Q$ factors under normal excitations are still limited. Here, we present the realizations of quasiBIC under normal excitation with a record $Q$ factor up to 18511 by engineering the symmetry properties and the number of the unit cells in all-dielectric metasurface platforms. The high- $Q$ quasi-BICs exhibit exceptionally high conversion efficiency for the third harmonic generation and even enable the second harmonic generation in $\mathrm{Si}$ metasurfaces. Such ultrasharp resonances achieved in this work may immediately boost the performances of BICs in a plethora of fundamental research and device applications, e.g., cavity QED, biosensing, nanolasing, and quantum light generations.

DOI: 10.1103/PhysRevLett.123.253901

High- $Q$ resonances are universal resources across different branches of physics, e.g., in acoustics, electronics, and electromagnetics, etc. They describe the high capacity of a physical system for storing energy. In nanophotonics, the high- $Q$ resonances directly enable serial enchanting developments in both fundamental research [1] and device applications [2]. The mainstream method of achieving high- $Q$ optical resonances at nanoscale is to employ total internal reflection that operates below the light line. For instance, the microcavities based on whispering gallery modes and 2D photonic band gap cavities exhibit extraordinary abilities of creating high- $Q$ resonances and therefore enable the observations and manipulations of exotic optical phenomena in a chip scale [3], such as parity-timesymmetric physics [4], broadband momentum transformation [5], exceptional points enhanced sensing [6], silicon Raman lasers [7], and efficient nonlinear harmonics generations [8]. On the other hand, the total internal reflection inevitably imposes an intrinsic challenge of realizing

Published by the American Physical Society under the terms of the Creative Commons Attribution 4.0 International license. Further distribution of this work must maintain attribution to the author(s) and the published article's title, journal citation, and DOI. efficient couplings between light from free space and the highly confined cavity modes despite such couplings being significantly improved with special techniques [9-11] and novel cavity engineering [12].

Bound states in the continuum (BICs) are wave solutions that are embedded in a radiative continuum but completely decouple from the continuum [13]. They can be regarded as the condition that discrete states forming Fano resonances become completely orthogonal to the radiative continuum [14,15], i.e., eigenmodes process infinite $Q$ factors. Therefore, these modes are inaccessible from external excitations belong to such a continuum. Because sitting in the radiative continuum, the BIC associated modes could be potentially accessible from the free space when they becomes quasi-BICs with minimized but perceptible interaction with the continuum. Specifically, triggering the transition from BICs to quasi-BICs accessible from the radiative continuum is of paramount importance for building practical devices that are able to harness the functionalities of BIC modes [16-19].

Recently, all-dielectric resonant metasurfaces are emerging as a versatile tool to realize quasi-BICs in finite structures associated with BICs under normal excitations by engineering the structure symmetry of the individual scattering units with Mie resonances [20,21]. Fano resonances originated from BICs achieved in such a low-loss 
TABLE I. Comparison of $Q$ factors measured under normal excitation conditions for all-dielectric metasurfaces.

\begin{tabular}{lccr}
\hline \hline Year & References & Wavelength $(\mathrm{nm})$ & $Q$ factor \\
\hline 2014 & {$[37]$} & 1376 & 483 \\
2016 & {$[38]$} & 1000 & 350 \\
2017 & {$[39]$} & 1500 & 300 \\
2017 & {$[29]$} & 1300 & 1011 \\
2018 & {$[40]$} & 1490 & 1946 \\
2018 & {$[32]$} & 825 & 2750 \\
2018 & {$[33]$} & 2320 & 150 \\
2018 & {$[26]$} & 5700 & 200 \\
2019 & {$[27]$} & 855 & 144 \\
2019 & This work & 1588 & 18511 \\
\hline \hline
\end{tabular}

flat platform have boosted a variety of applications that are in favor of high- $Q$ resonances and tight light confinements at nanoscale [22-25]. E.g., all-dielectric high- $Q$ metasurfaces have recently enabled the ultrasensitive detection of molecular fingerprints and hyperspectral imaging for biosensing without the utilization of spectrometers [26-28]. The high- $Q$ metasurfaces were also successfully employed to build novel flat light emitting devices via engineering of spontaneous emission [29,30], stimulated emission [31,32], and nonlinear harmonic generations [33-35].

In the experiments, the $Q$ factor consists of the radiative part $Q_{r}$ and nonradiative part $Q_{\mathrm{nr}}$ via $1 / Q=1 / Q_{r}+1 / Q_{\mathrm{nr}}$. (a)

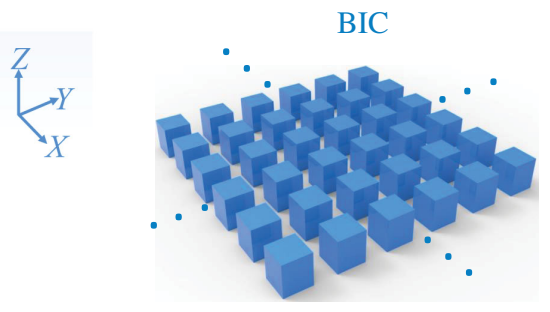

(d)
In particular, $Q_{\mathrm{nr}}$ covers surface roughness, structure disorder, the deviation of fabricated structure from the design, etc. To date, the highest $Q$ factors of the all-dielectric metasurfaces under normal excitation reported so far are rather low (a few thousand, see Table I) mostly due to the relatively low value of $Q_{\mathrm{nr}}$ in the optical domain and therefore have greatly hindered the exploration of new physics under strongly enhanced light-matter interaction as well as their potential device applications in nonlinear optics $[5,8,33-36]$. Leveraging by the significantly enhanced $Q_{\mathrm{nr}}\left(\sim 10^{5}\right)$, in this Letter, we have achieved high- $Q$ quasi-BICs with record $Q$ factors up to 18511 in all-dielectric metasurfaces by engineering the in-plane symmetry and the number of the unit cell simultaneously. As straightforward applications, we further demonstrate exceptionally efficient third harmonic generation (THG) and even observe the second harmonic generation (SHG) in high- $Q$ Si metasurfaces.

We consider symmetry protected BIC modes in $\mathrm{Si}$ blocks arranging in a square lattice, as shown in Fig. 1(a). Such a periodic metasurface supports a BIC with an infinite $Q$ factor at the $\Gamma$ point of the first Brillouin zone [see Figs. 1(b) and 1(d) for $S_{1}=0$ and $S_{2}=0 \mathrm{~nm}$ ]. The electromagnetic field distribution of the eigenmode, shown in the inset of Fig. 1(b), reveals the magnetic dipole (MD) nature of the BIC with its dipole moment along the $Z$ axis. In order to excite a high $Q$ factor MD resonance (c)

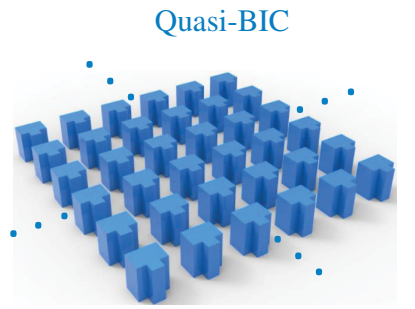

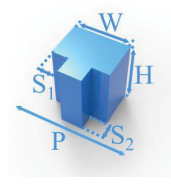

(e)
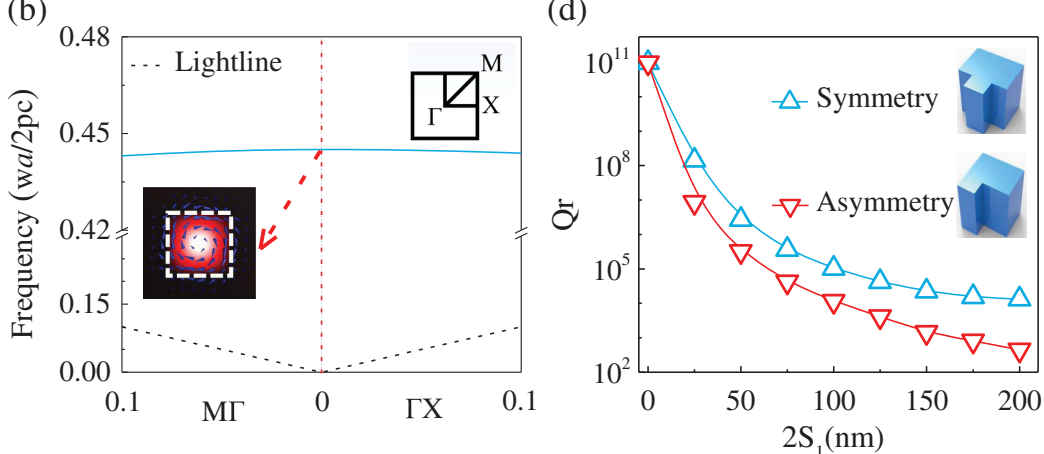

FIG. 1. Transition from BICs to quasi-BIC. (a) Periodic arrangement of Mie resonators supporting BICs $(W=400, H=500$, and $P=720 \mathrm{~nm}$ ). (b) Band structure related to the MD BIC mode. Inset: the electromagnetic field distribution of the BIC mode in a single unit, where the color code presents the amplitude of the magnetic field, the blue arrows indicate the in-plane electric field vectors, and the white square outlines the structure. (c) $N \times N$ Si blocks with symmetric defects that supports quasi-BICs. (d) Dependence of the $Q_{r}$ of quasi-BIC eigenmodes (infinite size) on the degrees of in-plane symmetry breaking for two types of symmetry breaking methods. (e) The transmission spectra of $9 \times 9$ Si blocks with symmetric defects excited by normally incident $x$-/ $y$-polarized plane waves from the $z$ direction $\left(S_{1}=60\right.$ and $\left.S_{2}=160 \mathrm{~nm}\right)$. The electromagnetic field distributions of the quasi-BIC and the one in the center unit cell at the resonance are shown by the insets. 
associated with the MD-BIC mode under normal excitations, symmetry breaking should be exploited to trigger the transition from BICs to the quasi-BIC modes [23-25] [see insets of Fig. 1(c)]. The smaller degree of symmetry breaking is, the higher $Q_{r}$ can be obtained [23-25]. Therefore, introduction of asymmetry as small as possible is crucial for achieving high- $Q$ quasi-BICs. The conventional way of symmetry breaking is simply removing material at one corner of the unit cell, which can be regarded as the asymmetric defect [see the lower inset of Fig. 1(d)] [38]. Alternatively, we propose to introduce symmetric defects by removing the same volume of material in the unit cell, as shown in the upper inset of Fig. 1(d). The symmetric defects still preserve the mirror symmetry of the structure respective to the $X Z$ plane. As clearly shown by the eigensolutions of the infinite metasurface, the reduction of $Q_{r}$ with the increase in the size $\left(2 \times S_{1}\right)$ of the symmetric defect is much slower than that of the asymmetric one [see Fig. 1(d)]. Therefore, the symmetric defect to the largest extent maintains the $Q_{r}$ from the MD-BIC meanwhile making them externally accessible as a quasi-BIC. The transmission of the metasurface manifests itself as a sharp clean transmission peak with the MD signature $\left(M_{z}\right)$ in the telecom band verified by the corresponding multipolar decomposition results [41].

It should be kept in mind that true BICs only exist when the metasurface is symmetric and infinite [see Fig. 1(a)]. However, in reality, the physical sizes of the metasurface, which consist of $N \times N$ Mie resonators arranged as an array, are finite $[13,48]$. For example, a typical finite $(9 \times 9)$ metasurface supporting high- $Q$ MD quasi-BICs under the excitation of a normally incident $y$-polarized plane wave is shown in Fig. 1(e) with the corresponding electromagnetic field distribution shown in the inset. The quasi-BIC is
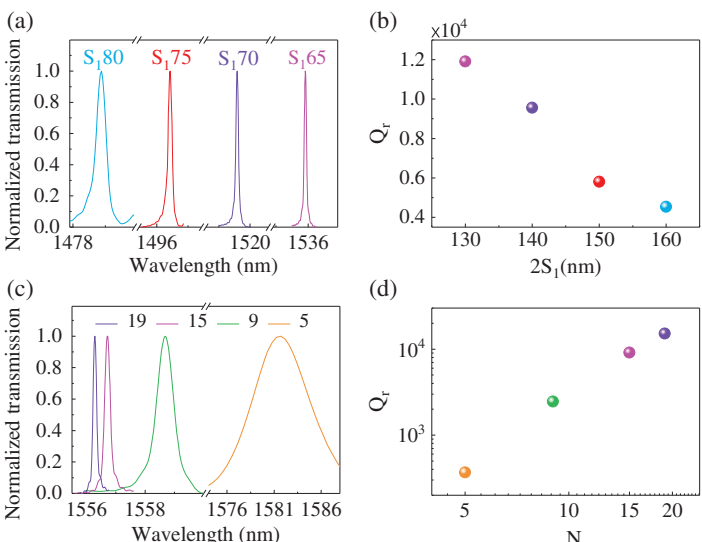

(d)

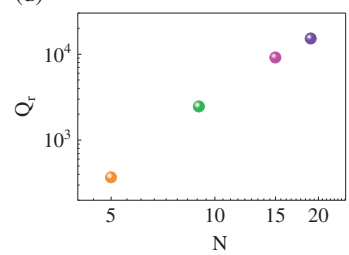

FIG. 2. Engineering the $Q_{r}$ and resonance wavelengths of the quasi-BIC in all-dielectric metasurfaces. (a) The calculated transmission spectra of $21 \times 21$ Si blocks with different defect sizes. (b) $Q_{r}$ extracted from (a) as a function of the defect size. (c) The calculated transmission spectra for $\mathrm{Si}$ metasurfaces $\left(S_{1}=60\right.$ and $S_{2}=160 \mathrm{~nm}$ ) with different array sizes. (d) $Q_{r}$ extracted from (c) as a function of the array size. polarization dependent and can be switched off when excited by the $x$-polarized plane wave due to the structural symmetry. The $Q_{r}$ of the MD resonance can be significantly increased accompanied with the redshifts of the quasi-BICs by reducing the volume removed from the Si block (defect size), as can be seen from the calculations of the $21 \times 21 \mathrm{Si}$ block array in Figs. 2(a) and 2(b). At the same time, the $Q_{r}$ can also be greatly boosted up together with the blueshift of resonances by increasing the number $N$ of the unit cell $[31,37,49]$, which shows a quadratic dependence on $N$, as presented in Figs. 2(c) and 2(d). These conclusions are similar to the case of the one-dimensional chain of resonators operating in the microwave regime [48]. As a result, engineering the structure symmetry and the number of the unit cell simultaneously provide efficient tuning knobs to control both $Q_{r}$ and the resonance wavelengths of the quasiBICs in all-dielectric metasurfaces. We note that the quasiBICs can also exist in the finite structure without introducing defects in the unit cell; however, such modes are hardly detectable from the scattering in the far field [41].

To experimentally verify our proposal, we have fabricated a serial $N \times N(N=5,9,15,19,21,27) T$-shape monocrystalline Si block array with distinct defect sizes on a quartz substrate [41]. Figure 3(a) presents a scanning electron beam (SEM) image of the fabricated metasurface, showing high-quality symmetric $T$-shape $\mathrm{Si}$ blocks in a square array. We first investigate the characteristics of quasi-BICs as a function of the defect size. The normalized transmission spectra of metasurfaces $(21 \times 21$ unit cells $)$ with different defect sizes are shown in Fig. 3(b) [41]. The sharp peaks in the transmission spectrum are the signatures of the quasi-BICs and they can be very well fitted by either Lorentzian or Fano shapes without any noticeable background or side peaks. Such sharp and clean resonances are particularly appealing in the biosensing application for reducing the cross talk when resolving the adjacent resonances [26-28]. With the reduction of the defect size, the spectral linewidth of the quasi-BIC clearly reduces together with redshifts of the resonance wavelength. The corresponding $Q$ factors of the quasi-BICs in Fig. 3(b) are plotted in Fig. 3(c), in which the experimental results are well predicted by our numerical simulations in Figs. 2(a) and 2(b). Based on the measured $Q$ factors and the simulated $Q_{r}$ [see Fig. 2(b)], $Q_{\mathrm{nr}}$ is deduced in the order of $10^{5}$, which validates the high quality of the fabricated metasurfaces [see the inset of Fig. 3(c)]. The other parameter that can be exploited to boost the $Q$ factor is the number of the unit cell, $N \times N$. The representative transmission spectra for metasurfaces with the same defects but different numbers of the unit cell are shown in Fig. 3(d). With the increase of the array size, the resonance wavelengths of metasurfaces blueshift together with significant enhancements of the $Q$ factors, which is also consistent with the features of the quasi-BICs calculated by the simulations in Figs. 2(c) and 2(d). It should be pointed out that the intrinsic fabrication uncertainty might result in 
(a)

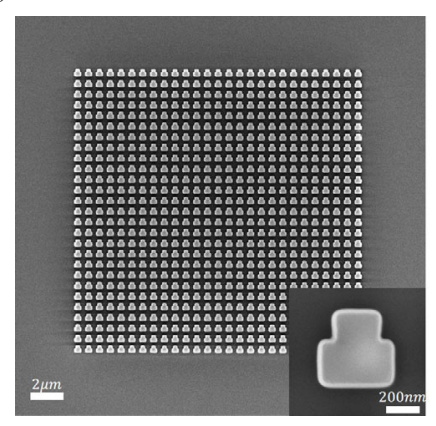

(b)

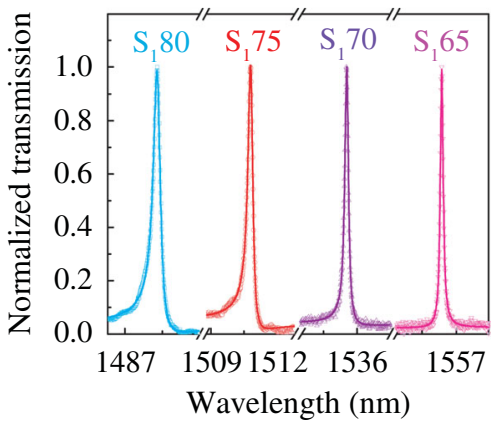

(c)

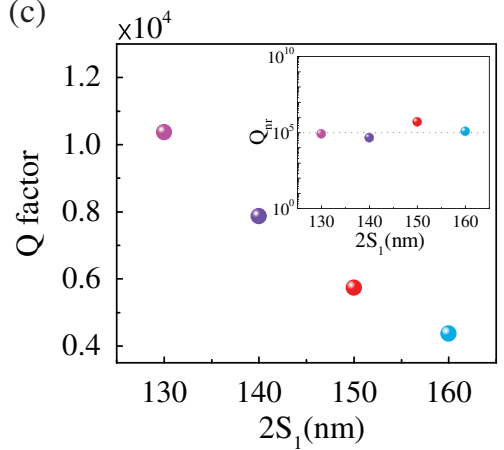

(d)

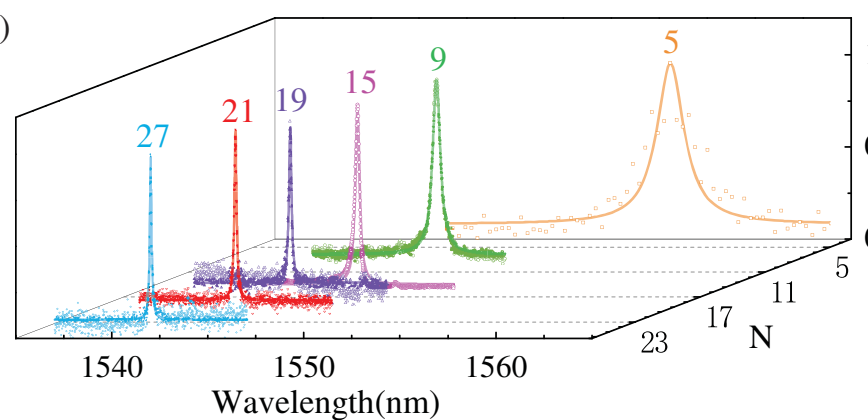

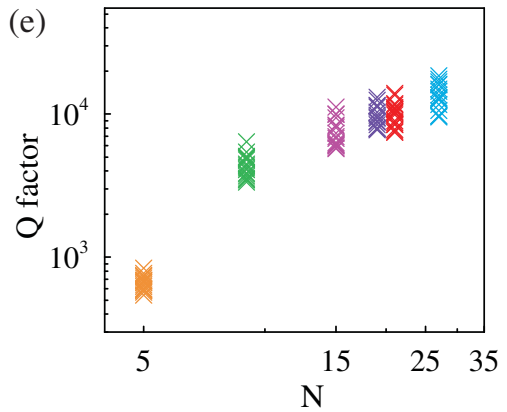

FIG. 3. Experimental demonstration of high- $Q$ quasi-BICs in Si metasurfaces. (a) SEM image of the fabricated device. (b) Transmission spectra of metasurfaces $(21 \times 21$ Si blocks array) with different defect sizes in the unit cell. (c) The extracted $Q$ factors from (b) as a function of the defect size. Inset: the calculated $Q_{\mathrm{nr}}$ for the devices. (d) Representative transmission spectra of Si metasurfaces with different array sizes. Here the targeted defect size is $S_{1}=65 \mathrm{~nm}$. (e) Statistics of measured $Q$ factors for all the fabricated $\mathrm{Si}$ metasurfaces with different array sizes.

a few nanometers shifting of resonant wavelength. In order to clearly show the relation between the $Q$ factor and the array size, the statistics of the measured $Q$ factors for all the fabricated devices with slightly different parameters are plotted as a function of $N$ as shown in Fig. 3(e). Both average and maximal values of the measured $Q$ factors dramatically increase with $N$. The maximal $Q$ factor measured in our samples is as high as 18511 from a $27 \times$ $27 \mathrm{Si}$ blocks array. Such a record high value, to the best of our knowledge, significantly outperforms any of the reported $Q$ factors of the existing metasurfaces under normal excitation, as listed in Table I. The $Q$ factor can be further improved by fabricating smaller defects with specialized process.

The optical harmonic generation (HG) is one of most promising applications of all-dielectric metasurfaces and it can be greatly enhanced by the electromagnetic resonances, e.g., the Mie and Fano resonances [33-35]. Based on the high- $Q$ all-dielectric platform realized above, we have investigated the HGs in the finite size metasurface by pumping at the resonant wavelength with picosecond pulses ( $\sim 5 \mathrm{ps)}$ [41]. It should be pointed out that we use the metasurface with $9 \times 9$ units for nonlinear optical measurement as we have a picosecond laser whose spectral linewidth is matched to that of the quasi-BIC resonance. The THG and SHG from our metasurface and (a)

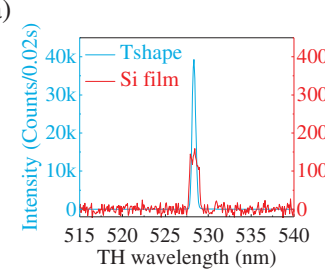

(b)

(c)

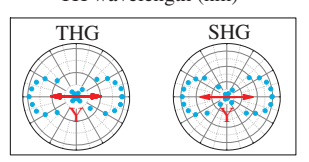

(e)

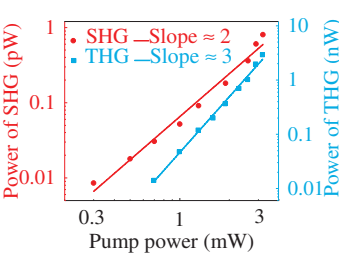

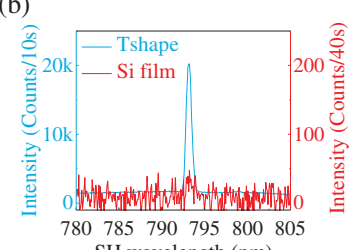

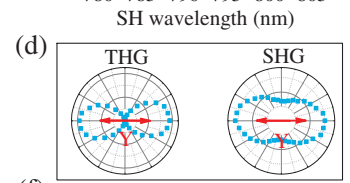

(f)

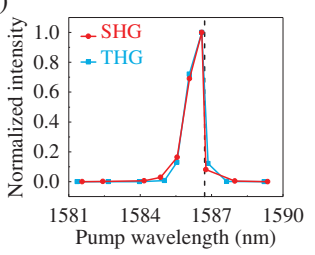

FIG. 4. THG/SHG generations in all-dielectric metasurfaces. Comparison of THG (a) and SHG (b) in all-dielectric metasurfacs and a reference $\mathrm{Si}$ thin film with the same thickness as the metasurfaces. (c) Dependence of THG/SHG on the polarization of the pumping laser. (d) The polar plots of the THG/SHG signals (fixing the pumping at $y$ polarization). (e) Power dependences of SHG/THG in logarithmic scale, showing quadratic and cubic power scalings, respectively. (f) Dependences of THG/SHG on the pumping wavelength. 
an un-patterned Si film are compared in Figs. 4(a) and 4(b). By pumping at the resonance using a picosecond pulsed laser, the THG signal is more than 5 orders of magnitude stronger than that from the bare Si film with the same thickness as the metasurface. Counterintuitively, pronounced SHG, generally absent in Si due to its centrosymmetric crystallography, is also observed in our $\mathrm{Si}$ metasurface while the SHG from the reference $\mathrm{Si}$ film is barely seen. The pronounced SHG from our metasurfaces can be attributed to the combination of enhancements of local field from high- $Q$ resonances and the optical nonliearity from the etched surfaces of Si. The links between the enhanced THG/SHG and the quasi-BIC can be seen by varying the polarization of the pumping laser. As shown in Fig. 4(c), the intensities THG and SHG reach to the maximal values when the pumping laser is $y$ polarized, which is consistent with polarization dependence of the transmission spectra in Fig. 1(e). The analysis of the polarizations of THG and SHG confirms that the SHG are governed by symmetry-breaking-induced nonlinearity at a surface [8], while the THG are dominated by the bulk nonlinear response of $\mathrm{Si}$, as shown in Fig. 4(d). The THG and SHG are further verified by the power-dependent double logarithmic plots, showing characteristic cubic and quadratic power scaling respectively, as shown in Fig. 4(e). We note the normalized THG conversion efficiency of $1.4 \times 10^{-8} / \mathrm{W}^{2}$ in our work is at least 5 orders of magnitude higher than state-of-the-art achieved in $\mathrm{Si}$ metasurfaces, as shown in Table S1 [41]. The enhancements of THG/SHG due to the resonances of the metasurface is further visualized by the dependence of HG on the pumping wavelength, shown in Fig. 4(f). When scanning the wavelength of the pumping laser across the quasi-BIC, both THG and SHG reach to the maximal values at the resonance wavelength and dramatically reduced under the off-resonant conditions. The simultaneous enhancements of both THG and SHG unequivocally reveal the great potential of our high- $Q$ metasurfaces for nonlinear optics. In particular, by scaling the resonances beyond $2.1 \mu \mathrm{m}$ to avoid two-photon and free-carrier absorption effects in $\mathrm{Si}$ material at $1550 \mathrm{~nm}$, efficient high-order harmonic generations could be immediately expected in our devices [33].

In summary, we have managed to boost the $Q$ factors of all metasurfaces under normal excitation via engineering the quasi-BIC. A quasi-BIC with a record $Q$ factor as high as 18511 under normal excitation was experimentally demonstrated in a $\mathrm{Si}$ metasurface $\left(19 \times 19 \mu \mathrm{m}^{2}\right)$. Our design and fabrication are versatile so that it can be easily scaled to different wavelengths, e.g., mid-IR for sensing and adopted with active material, e.g., GaAs for coherent light generation. To further boost the $Q$ factor of the quasi-BIC originated from BICs, reducing intrinsic disorder in the fabrication or employing quasi-BIC originated from topologically protected BICs could be explored in future investigations [50,51]. The advent of high- $Q$ BICs in metasurfaces could immediately bring fascinating opportunities both in fundamental physics and device applications, from magnetic cavity QED, coherent and quantum light generations, to ultrasensitive biosensing and high-harmonic generations, etc., in the popular flat optics platform.

This research was supported by National Key R\&D Program of China (2018YFA0306100), the National Natural Science Foundation of China (11874437, 11674130, 91750110, 11674110, 11874020, 11761131001), Guangzhou Science and Technology Project (201805010004), the Natural Science Foundation of Guangdong (2018B030311027, 2016A030306016, 2016TQ03X981), the Pearl River Nova Program of Guangzhou (201806010040), the Fundamental Research Funds for the Central Universities and the national supercomputer center in Guangzhou. We thank Lidan Zhou, Lin Liu for the assistance in the sample fabrication, and Fan $\mathrm{Li}$, Zhaohui Li for loaning the optical spectrum analyzer.

*yi.xu@osamember.org

†liujin23@mail.sysu.edu.cn

[1] K. J. Vahala, Nature (London) 424, 839 (2003).

[2] F. Vollmer and L. Yang, Nanophotonics 1, 267 (2012).

[3] Y. Akahane, T. Asano, B. S. Song, and S. Noda, Nature (London) 425, 944 (2003).

[4] B. Peng, Ş. K. Özdemir, F. Lei, F. Monifi, M. Gianfreda, G. L. Long, S. Fan, F. Nori, C. M. Bender, and L. Yang, Nat. Phys. 10, 394 (2014).

[5] X. Jiang, L. Shao, S.-X. Zhang, X. Yi, J. Wiersig, L. Wang, Q. Gong, M. Lončar, L. Yang, and Y.-F. Xiao, Science 358, 344 (2017).

[6] W. Chen, Ş. K. Özdemir, G. Zhao, J. Wiersig, and L. Yang, Nature (London) 548, 192 (2017).

[7] Y. Takahashi, Y. Inui, M. Chihara, T. Asano, R. Terawaki, and S. Noda, Nature (London) 498, 470 (2013).

[8] X. Zhang, Q.-T. Cao, Z. Wang, Y.-x. Liu, C.-W. Qiu, L. Yang, Q. Gong, and Y.-F. Xiao, Nat. Photonics 13, 21 (2019).

[9] T. G. Tiecke, K. P. Nayak, J. D. Thompson, T. Peyronel, N. P. de Leon, V. Vuletić, and M. D. Lukin, Optica 2, 70 (2015).

[10] K. Srinivasan, P. E. Barclay, M. Borselli, and O. Painter, Phys. Rev. B 70, 081306(R) (2004).

[11] Y. Ren, R. Zhang, C. Ti, and Y. Liu, Optica 3, 1205 (2016).

[12] Y. F. Xiao, C.-L. Zou, Y. Li, C.-H. Dong, Z.-F. Han, and Q. Gong, Front. Optoelectron. China 3, 109 (2010).

[13] C. W. Hsu, B. Zhen, A. D. Stone, J. D. Joannopoulos, and M. Soljačić, Nat. Rev. Mater. 1, 16048 (2016).

[14] M. F. Limonov, M. V. Rybin, A. N. Poddubny, and Y. S. Kivshar, Nat. Photonics 11, 543 (2017).

[15] A. E. Miroshnichenko, S. Flach, and Y.S. Kivshar, Rev. Mod. Phys. 82, 2257 (2010).

[16] S. Fan and J. D. Joannopoulos, Phys. Rev. B 65, 235112 (2002).

[17] J. Lee, B. Zhen, S.-L. Chua, W. Qiu, J. D. Joannopoulos, M. Soljačić, and O. Shapira, Phys. Rev. Lett. 109, 067401 (2012).

[18] Y. Zhang, A. Chen, W. Liu, C. W. Hsu, B. Wang, F. Guan, X. Liu, L. Shi, L. Lu, and J. Zi, Phys. Rev. Lett. 120, 186103 (2018). 
[19] S. Dai, L. Liu, D. Han, and J. Zi, Phys. Rev. B 98, 081405(R) (2018).

[20] I. Staude and J. Schilling, Nat. Photonics 11, 274 (2017).

[21] K. Koshelev, A. Bogdanov, and Y. Kivshar, Sci. Bull. 64, 836 (2019).

[22] Y. Yang, C. Peng, Y. Liang, Z. Li, and S. Noda, Phys. Rev. Lett. 113, 037401 (2014).

[23] K. Koshelev, S. Lepeshov, M. Liu, A. Bogdanov, and Y. Kivshar, Phys. Rev. Lett. 121, 193903 (2018).

[24] Y. He, G. Guo, T. Feng, Y. Xu, and A. E. Miroshnichenko, Phys. Rev. B 98, 161112(R) (2018).

[25] A. S. Kupriianov, Y. Xu, A. Sayanskiy, V. Dmitriev, Y. S. Kivshar, and V. R. Tuz, Phys. Rev. Applied 12, 014024 (2019).

[26] A. Tittl, A. Leitis, M. Liu, F. Yesilkoy, D.-Y. Choi, D. N. Neshev, Y.S. Kivshar, and H. Altug, Science 360, 1105 (2018).

[27] F. Yesilkoy, E. R. Arvelo, Y. Jahani, M. Liu, A. Tittl, V. Cevher, Y. Kivshar, and H. Altug, Nat. Photonics 13, 390 (2019).

[28] A. Leitis, A. Tittl, M. Liu, B. H. Lee, M. B. Gu, Y. S. Kivshar, and H. Altug, Sci. Adv. 5, eaaw2871 (2019).

[29] S. Yuan, X. Qiu, C. Cui, L. Zhu, Y. Wang, Y. Li, J. Song, Q. Huang, and J. Xia, ACS Nano 11, 10704 (2017).

[30] S. Liu et al., Nano Lett. 18, 6906 (2018).

[31] A. Kodigala, T. Lepetit, Q. Gu, B. Bahari, Y. Fainman, and B. Kanté, Nature (London) 541, 196 (2017).

[32] S. T. Ha, Y. H. Fu, N. K. Emani, Z. Pan, R. M. Bakker, R. Paniagua-Domínguez, and A. I. Kuznetsov, Nat. Nanotechnol. 13, 1042 (2018).

[33] H. Liu, C. Guo, G. Vampa, J. L. Zhang, T. Sarmiento, M. Xiao, P. H. Bucksbaum, J. Vučković, S. Fan, and D. A. Reis, Nat. Phys. 14, 1006 (2018).

[34] L. Xu et al., Adv. Sci. 6, 1802119 (2019).

[35] K. Koshelev, Y. Tang, K. Li, D.-Y. Choi, G. Li, and Y. Kivshar, ACS Photonics 6, 1639 (2019).

[36] L. Carletti, K. Koshelev, C. De Angelis, and Y. Kivshar, Phys. Rev. Lett. 121, 033903 (2018).
[37] Y. Yang, I. I. Kravchenko, D. P. Briggs, and J. Valentine, Nat. Commun. 5, 5753 (2014).

[38] S. Campione et al., ACS Photonics 3, 2362 (2016).

[39] Z. F. Sadrieva, I. S. Sinev, K. L. Koshelev, A. Samusev, I. V. Iorsh, O. Takayama, R. Malureanu, A. A. Bogdanov, and A. V. Lavrinenko, ACS Photonics 4, 723 (2017).

[40] C. Cui et al., ACS Photonics 5, 4074 (2018).

[41] See Supplemental Material at http://link.aps.org/ supplemental/10.1103/PhysRevLett.123.253901 for electromagnetic multipolar decomposition analysis, which includes Ref. [42]; for the coupled electric and magnetic dipole approximation method, which includes Refs. [43,44]; for sample fabrication, linear transmission, and optical setup; for estimation of the THG conversion efficiency, which includes Refs. [45,46,47].

[42] A. B. Evlyukhin, C. Reinhardt, and B. N. Chichkov, Phys. Rev. B 84, 235429 (2011).

[43] C. F. Bohren and D. R. Huffman, Absorption and Scattering of Light by Small Particles (John Wiley and Sons, New York, 2008).

[44] G. W. Mulholland, C. F. Bohren, and K. A. Fuller, Langmuir 10, 2533 (1994).

[45] G. Grinblat, Y. Li, M. P. Nielsen, R. F. Oulton, and S. A. Maier, ACS Nano 11, 953 (2017).

[46] M. R. Shcherbakov et al., Nano Lett. 14, 6488 (2014).

[47] Y. Yang, W. Wang, A. Boulesbaa, I. I. Kravchenko, D. P. Briggs, A. Puretzky, D. Geohegan, and J. Valentine, Nano Lett. 15, 7388 (2015).

[48] Z. F. Sadrieva, M. A. Belyakov, M. A. Balezin, P. V. Kapitanova, E. A. Nenasheva, A. F. Sadreev, and A. A. Bogdanov, Phys. Rev. A 99, 053804 (2019).

[49] E. N. Bulgakov and A. F. Sadreev, Phys. Rev. A 97, 033834 (2018).

[50] B. Zhen, C. W. Hsu, L. Lu, A. D. Stone, and M. Soljačić, Phys. Rev. Lett. 113, 257401 (2014).

[51] J. Jin, X. Yin, L. Ni, M. Soljačić, B. Zhen, and C. Peng, Nature (London) 574, 501 (2019). 